\title{
BEH Boson properties from CMS results
}

\author{
Paolo Checchia ${ }^{1, a}$ on behalf of CMS Collaboration \\ ${ }^{1}$ INFN sezione di Padova, Padova, Italy.
}

\begin{abstract}
The most relevant results on Higgs sector from CMS are presented. The status of the measurements of the Higgs Boson properties after the complete analysis of Run I dataset and an overview of the results obtained with the limited luminosity delivered at $13 \mathrm{TeV}$ in 2015 are given. Implications of the results and future perspectives are also briefly discussed.
\end{abstract}

\section{Introduction}

The discovery of the Brout-Engler-Higgs (BEH) boson [1-5], at CERN by the ATLAS[6] and CMS[7] experiments has been one of the most relevant results in particle physics. After several decades of searches for the BEH (hereafter simply $H$ ) particle mainly at LEP, Tevatron and LHC, the observation of a new state in the mass region still not excluded, is a step forward in the comprehension of the mechanism capable to generate the mass of elementary particles. This mechanism, called "BEH" or "Higgs" mechanism, is a crucial part of the standard model (SM) of particle physics and the discovery of the $H$ particle with a mass compatible with the SM expectations is an additional success of the model. However the discovered particle could be the lightest state of a more complex Higgs sector as foreseen by several extensions of the SM, including supersymmetric models [8]. To establish the actual nature of the particle it is necessary to provide precision measurements of its properties, and this is one of the goals of the LHC experiments in the near future.

\section{Status of searches before the discovery}

The legacy of LEP and SLC SM precision measurements [9], and of LEP, Tevatron and LHC direct searches for the $H$ boson is contained in Fig. 1. In the SM, several physical observables can be predicted by calculating the effect of radiative corrections that depend on the Higgs boson mass. The $\Delta \chi^{2}$ as obtained from the SM fit to the precision measurements is superimposed to the regions excluded by direct searches. It is evident that the fit prefers a low value for the Higgs boson mass. In addition, only a very narrow window in the mass range remained not excluded by direct searches.

\section{Expected properties of the SM Higgs boson}

Within the standard model all Higgs boson properties depend only on its mass. 


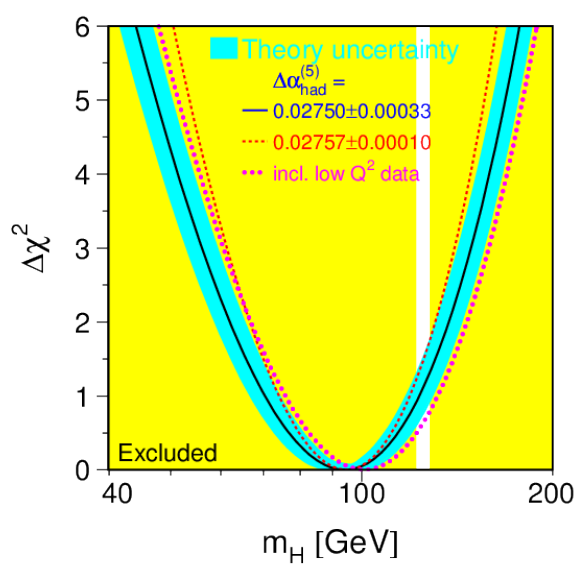

Figure 1. The status of the $\Delta \chi^{2}$ as obtained from the SM fit to the precision measurements superimposed to the direct searches for the SM Higgs boson. The blue band represents an estimate of the theoretical error. This figure is taken from Ref. [9].
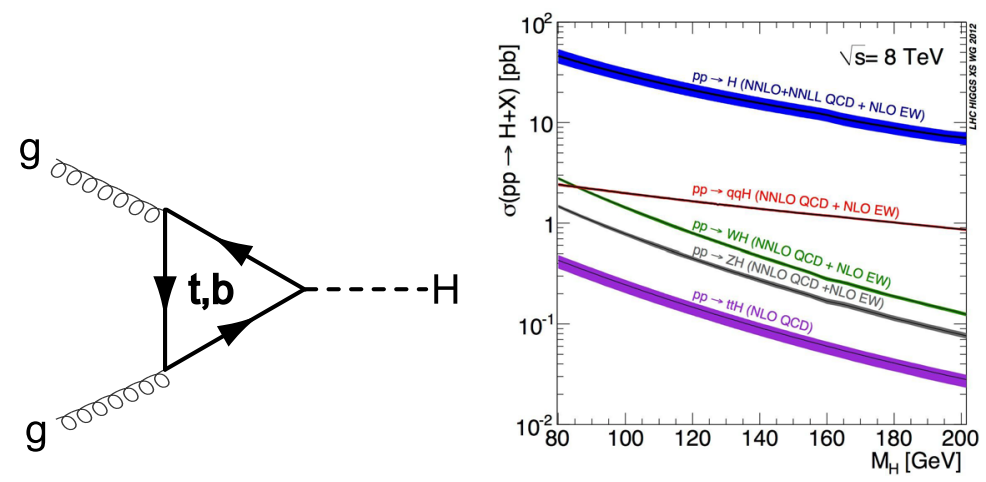

Figure 2. Gluon-gluon fusion (left) and production cross sections (right).

At a hadron collider the SM Higgs boson production is largely dominated by the two gluons collision (Fig. 2). However, other production mechanisms such as vector boson fusion (VBF) and associated production $\left(V H\right.$, where $V=W^{ \pm}$or $Z$, or $t \bar{t} H$ ) can play a relevant role since the presence of other energetic particles in the final state can be useful in reducing the background (Fig. 3).

In the low mass region where the Higgs particle cannot decay to on-shell gauge bosons, the total decay with $\left(\Gamma_{H}\right)$ is expected to be very narrow. In that region the decay is dominated by b-quark pairs but several channels have a branching ratio with a reasonable possibility to be measured (Fig 4).

\footnotetext{
a e-mail: paolo.checchia@pd.infn.it
} 

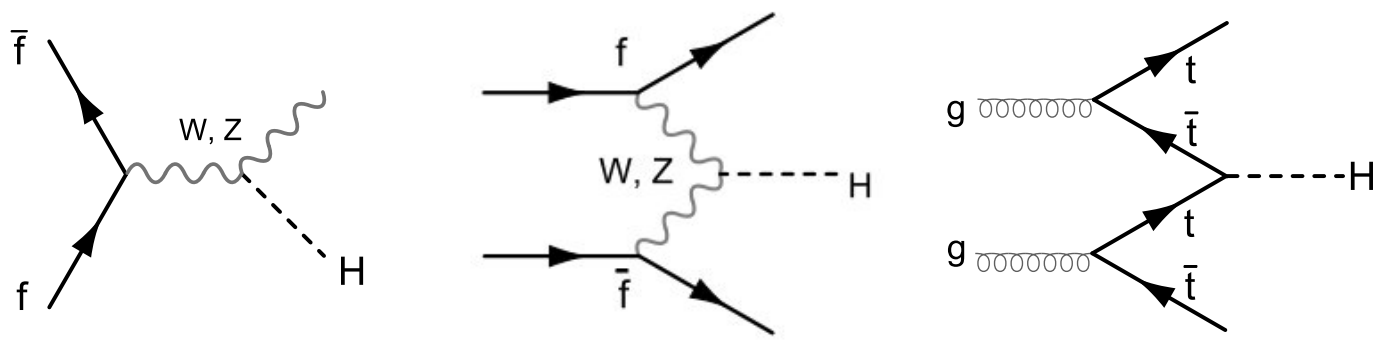

Figure 3. Higgs-strahlung or $V H$ (left), vector boson fusion or VBF (centre) and top-fusion (right) production processes.
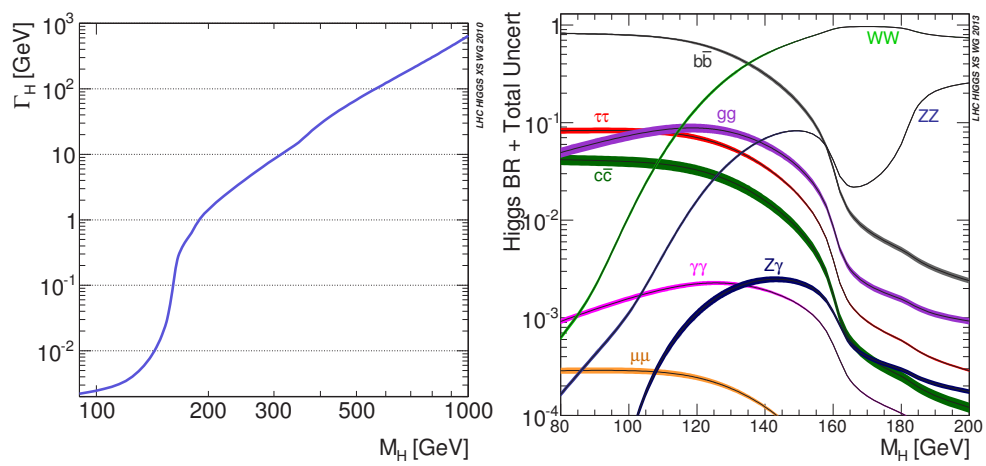

Figure 4. Total decay width (left) and decay branching fractions to the most relevant final states (right) as a function of the SM Higgs boson mass.

\section{The Higgs boson properties after LHC Run 1}

\subsection{The mass}

The measurement of the Higgs boson mass $\left(m_{H}\right)$, necessary to quantify the SM predictions on the particle properties, relies on the same final states relevant for the discovery: the $H \rightarrow Z Z^{*} \rightarrow 4 \ell$ $(\ell=e, \mu)$ and $H \rightarrow \gamma \gamma$ since for both channels the detector resolution allows to obtain a narrow peak on the reconstructed invariant mass.

In the case of the four lepton final state [10], both lepton flavours are well measured and in addition the $Z \rightarrow 2 \ell \gamma^{*} \rightarrow 4 \ell$ decay can be used to cross check the calibration (Fig. 5). This channel offers additional advantages since the background is small and it is also possible to enhance the signal significance by using discriminant variables based on the event kinematics. The measured mass is $m_{H}=125.6 \pm 0.4$ (stat) \pm 0.2 (syst) GeV. The statistical error dominates and the systematic uncertainty is mainly due to energy-momentum scale.

Also the diphoton decay channel [11] produces a narrow peak, given the excellent CMS electromagnetic calorimeter, but it has to deal with a large background. Therefore, to improve the analysis, the data are subdivided in categories with different invariant mass resolutions and different signal-tobackground ratios. It is then possible to visualize the signal by summing all the categories with a weight proportional to signal/signal plus background ratio: $w=S /(S+B)$. The mass measurement is 
$m_{H}=124.70 \pm 0.31$ (stat) \pm 0.15 (syst) GeV. Also in this case the error is dominated by the statistical contribution, with a dominant systematic uncertainty due to the photon energy scale.
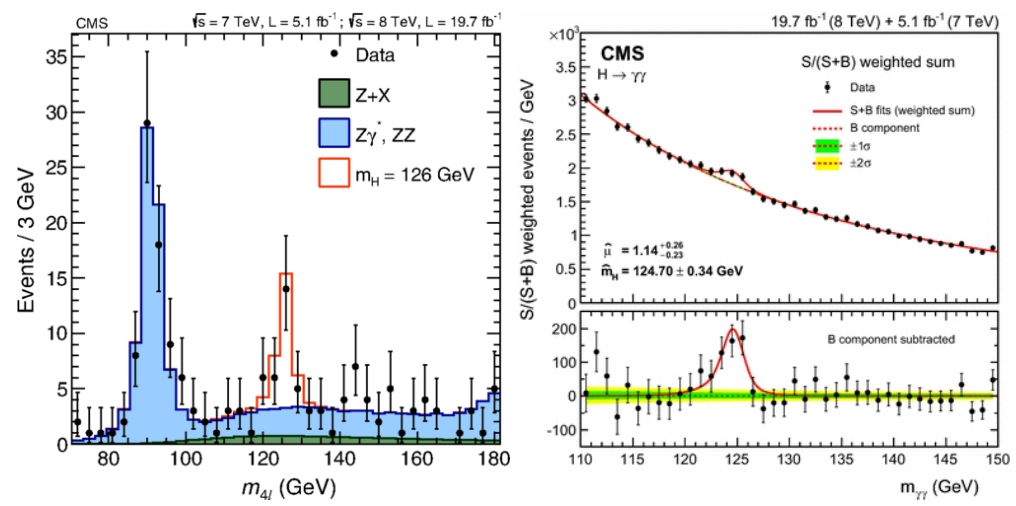

Figure 5. Invariant mass distribution of four lepton final state [10](left). Weighted invariant mass distribution of diphoton final state (see text) together with the background-subtracted distribution (right).

The two measurements are compatible within $\sim 1.6 \sigma$. They have been combined giving the final CMS determination of the Higgs boson mass after RUN 1: $m_{H}=$ $125.02_{-0.27}^{+0.26}$ (stat) ${ }_{-0.15}^{+0.14}$ (syst) $\mathrm{GeV}$ [12]. Furthermore, the CMS results have been combined with the ATLAS mass measurements of the Higgs boson obtaining [13]: $m_{H}=125.09 \pm$ 0.21 (stat) \pm 0.11 (syst) GeV.

\subsection{Spin-parity}

The new particle with a mass of $\sim 125 \mathrm{GeV}$ is a Higgs boson if it can be proven to be a scalar state. It is then necessary to show that the hypothesis $J^{P}=0^{+}$is the most probable. The direct decay into the diphoton final state excludes the spin 1 hypothesis. Other hypotheses are compared to the SM $0^{+}$ hypothesis by considering the kinematic properties of several final state decays. In particular, in the $H \rightarrow Z Z^{*} \rightarrow 4 \ell$ final state, the distributions of the decay angles $\Phi, \theta_{1}$ and $\theta_{2}$ (Fig. 6) bring information on the parity of the initial state.

A signal plus background likelihood function $\mathcal{L}_{\mathcal{J}^{\mathcal{P}}}$, for a given $J^{P}$ hypothesis, is defined by combining all the discriminant observables. The test statistic to compare the $J^{P}$ hypothesis with the $\mathrm{SM}\left(0^{+}\right)$hypothesis is $q=-2 \ln \left(\mathcal{L}_{J^{P}} / \mathcal{L}_{0^{+}}\right)$. The expected $q$ distributions for $0^{-}$hypothesis and SM Higgs boson are shown in the right panel of Fig. 6. Defining the cumulative probability $P_{J^{P}}=P(q>$ $\left.q_{o b s} \mid J^{P}+b k g\right)$, from the ratio $\mathrm{CL}_{s}=P_{0^{-}} / P_{0^{+}}$the pseudoscalar hypothesis is excluded at $99.9 \%$ C.L..

Other spin-parity hypotheses have been considered but they are all disfavoured and hence the discovered particle is very likely a scalar state.

\subsection{WW channel}

For a Higgs boson with a mass of about $125 \mathrm{GeV}$, the SM foresees that the $H \rightarrow W W$ decay channel has the second largest branching ratio. However, only the final states with double $W \rightarrow \ell v$ decay can be easily distinguished from background, given the presence of several relevant contributions. This decay chain has two neutrinos in the final state and hence it is not possible to fully reconstruct the invariant mass. Therefore, the signal must be detected as an excess of events over the expected 

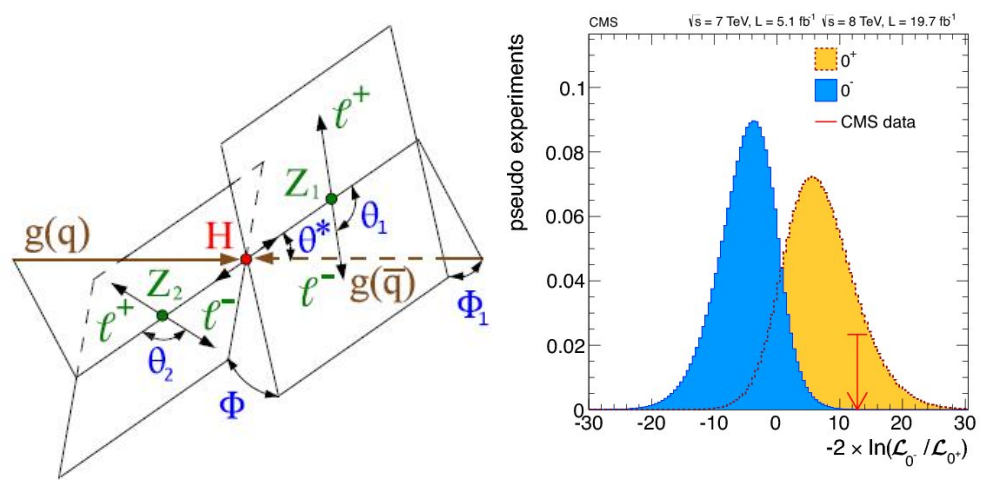

Figure 6. Illustration of the production and decay of a particle $H$. The angles $\theta^{*}, \Phi_{1}$ and $\Phi$ are given in the $H$ rest frame, and $\theta_{1}, \theta_{2}$ in the $Z_{1}, Z_{2}$ rest frames, respectively (left). Distributions of the test statistics $q=-2 \ln \left(\mathcal{L}_{J^{P}} / \mathcal{L}_{0^{+}}\right)$. The distribution of the SM Higgs boson $\left(0^{+}\right)$is shown in the yellow histogram while the distribution for the pseudoscalar hypothesis is represented by the blue histogram. The observed value is indicated by the red arrow (right).

background in regions of properly defined kinematic quantities. The azimuthal angle $\Delta \phi_{\ell \ell}$ between the leptons, their mass $m_{\ell \ell}$ and the transverse mass $m_{T}$ are used as discriminant kinematic variables [14]. The last is defined as $m_{T}=\sqrt{2 p_{T}^{\ell \ell} E_{T}^{\text {miss }}\left(1-\cos \Delta \phi_{\ell \ell, E_{T}^{m i s s}}\right)}$ and its distribution is shown in Fig.7. To optimise the signal/background ratio, the events are grouped in categories based on the lepton flavour and on the number of jets in the final state. There is a clear excess of events with respect to the background only hypothesis. From a $\left(m_{T}, m_{\ell \ell}\right)$ template fit the observed excess is quantified in $4.3 \sigma$, while the expected signal significance for a SM Higgs boson with $m_{H}=125.6 \mathrm{GeV}$ is $5.8 \sigma$.

\subsection{Fermionic channels}

Since in the SM the Yukawa coupling is proportional to the fermion mass, the $H \rightarrow \tau \tau$ decay has a large branching ratio compared to the other leptons. On the other hand, in this channel the mass reconstruction is problematic given the presence of neutrinos from the $\tau$ decay in the final state. The background from $Z \rightarrow \tau \tau$ can be separated on the basis of the difference in mass only once a dedicated mass reconstruction is implemented. After LHC RUN 1 CMS observes a significance of $3.2 \sigma$ with respect to the background only hypothesis to be compared with an expected significance of $3.7 \sigma$.

The inclusive $H \rightarrow b \bar{b}$ production, despite its high rate, is difficult to detect given the huge QCD $b \bar{b}$ background. For this reason CMS limits the analysis to the associate production $(V H)[15]$ and to VBF production [16]. For the former, the presence of additional high $p_{T}$ objects from gauge boson decay, allows to reduce the background and also to implement an efficient trigger for the events. An excess of events over the background-only hypothesis has been observed, corresponding to a significance of 2.1 standard deviations ( 2.1 expected). This result, combined with the $H \rightarrow \tau \tau$ channel, gives an overall significance of $3.8 \sigma$ [17] that constitutes an evidence that the particle at $125 \mathrm{GeV}$ is coupled to fermions as predicted for the Higgs boson in SM. Concerning the VBF production, CMS observes a deviation with respect to the background only hypothesis with a significance of $2.2 \sigma$, larger than expectations from SM predictions $(0.8 \sigma)$.

In SM, the coupling to the top quark is the largest Yukawa coupling of the Higgs boson to a fermion. Therefore, in absence of direct decay, the $t \bar{t} H$ production is the experimental tool to measure the coupling between top quark and Higgs boson (Fig. 3). To obtain the best sensitivity, the events are 

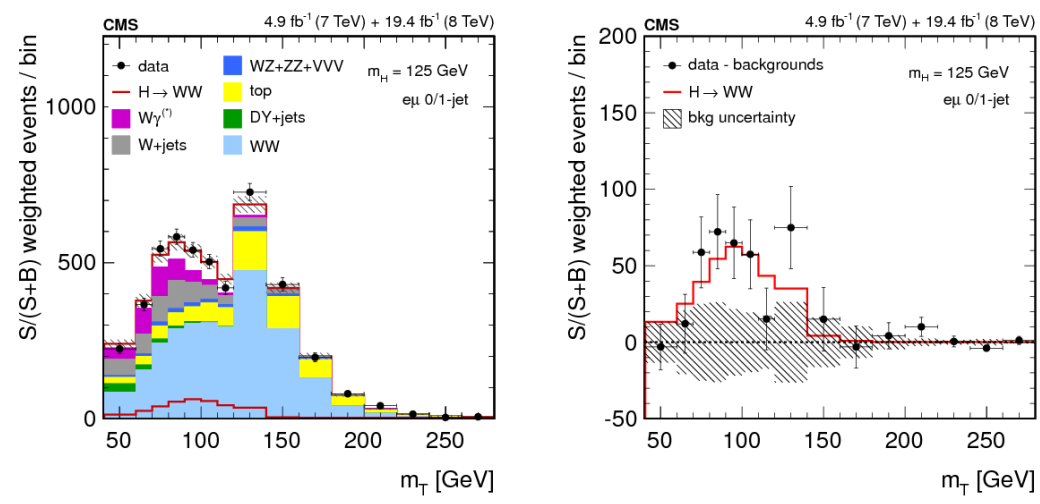

Figure 7. Distributions of $m_{T}$ in the $e \mu$ final state. The observed data (black points) are superimposed to the simulated events for signal+background and signal alone (left). The background-subtracted data with the fitted signal component (right).

divided in several categories depending on the top and Higgs boson decay. After RUN 1, CMS observes [18] a deviation with respect to the background only hypothesis which is higher than expected: $3.4 \sigma$ to be compared to an expectation of $1.2 \sigma$. The deviation with respect to the background plus $\mathrm{SM} t \bar{t} H$ signal is about two standard deviations. A new method based on matrix elements [19], limited to $H \rightarrow b \bar{b}$ decay, does not produce evidence of a signal, within the analysed statistics $\left(19.5 \mathrm{fb}^{-1}\right)$, but it represents a promising strategy for future precise measurements of the top Yukawa coupling.

\subsection{Couplings}

In order to understand more on the nature of the discovered particle, a direct comparison with the SM expectations is convenient. Given the vacuum expectation value (VEV) $v=\left(\sqrt{2} G_{F}\right)^{-1 / 2} \approx 246 \mathrm{GeV}$, the SM couplings to vector bosons are $g_{H V V}=\frac{2 m_{V}^{2}}{v}$ while the Yukawa couplings to fermions are proportional to the fermion masses: $g_{H f f}=\frac{m_{f}}{v}$. It is then useful to introduce the parameters given in Table 1 [20] to compare deviations from the SM predictions. When the coupling is not direct as in gluon fusion production or in diphoton decay, the coupling modifiers are calculated in terms of the direct parameters: $\kappa_{g}=\kappa_{g}\left(\kappa_{b}, \kappa_{t}, m_{H}\right), \kappa_{\gamma}=\kappa_{\gamma}\left(\kappa_{b}, \kappa_{t}, \kappa_{W}, m_{H}\right)$.

The parameters obtained by fitting all RUN 1 data are shown in Fig. 8 [12]. The combined signal strength is $\mu=1.0 \pm 0.14$ in perfect agreement with SM expectations for $m_{H}=125 \mathrm{GeV}$. The compatibility with the SM is evident for most of the results, with a few exceptions as for the top production mentioned above. In addition, the so-called "custodial symmetry" [21] has been tested, that foresees equal coupling modifiers for gauge bosons. The combined analysis of all channels gives $\lambda_{W Z}=0.91_{-0.12}^{+0.14}$ and then the assumption $\lambda_{W Z}=1$ is reasonable. As a consequence, further tests based on the hypothesis $\kappa_{W}=\kappa_{Z}=\kappa_{V}$ are exploited. In particular, with additional assumptions, including the hypothesis of equal coupling modifiers for fermions $\left(\kappa_{f}\right)$, a test of coupling to fermions and vector 
Table 1. Definition of some parameters to compare deviations from SM predictions.

\begin{tabular}{|l|c|c|}
\hline Name & meaning & symbol \\
\hline Signal strength & $\frac{\sigma(B R)}{\sigma^{S M}\left(B R^{S M}\right)}$ & $\mu$ \\
coupling modifiers & $\frac{g_{H X X}}{g_{H X X}^{S M}}$ & $\kappa_{X}$ \\
total width modifier & $\frac{\Gamma_{t o t}}{\Gamma_{t o t}^{S M}}$ & $\kappa_{H}^{2}$ \\
ratio of coupling modifiers & $\frac{K_{X}}{K_{Y}}$ & $\lambda_{X Y}$ \\
\hline
\end{tabular}

bosons is performed. In Fig. 9 the Confidence Level contours of the fit are shown in the $\kappa_{f}, \kappa_{V}$ plane. The best fit results are $\kappa_{V}=1.01 \pm 0.07, \kappa_{f}=0.89_{-0.13}^{+0.14}$, fully compatible with the SM expectations.
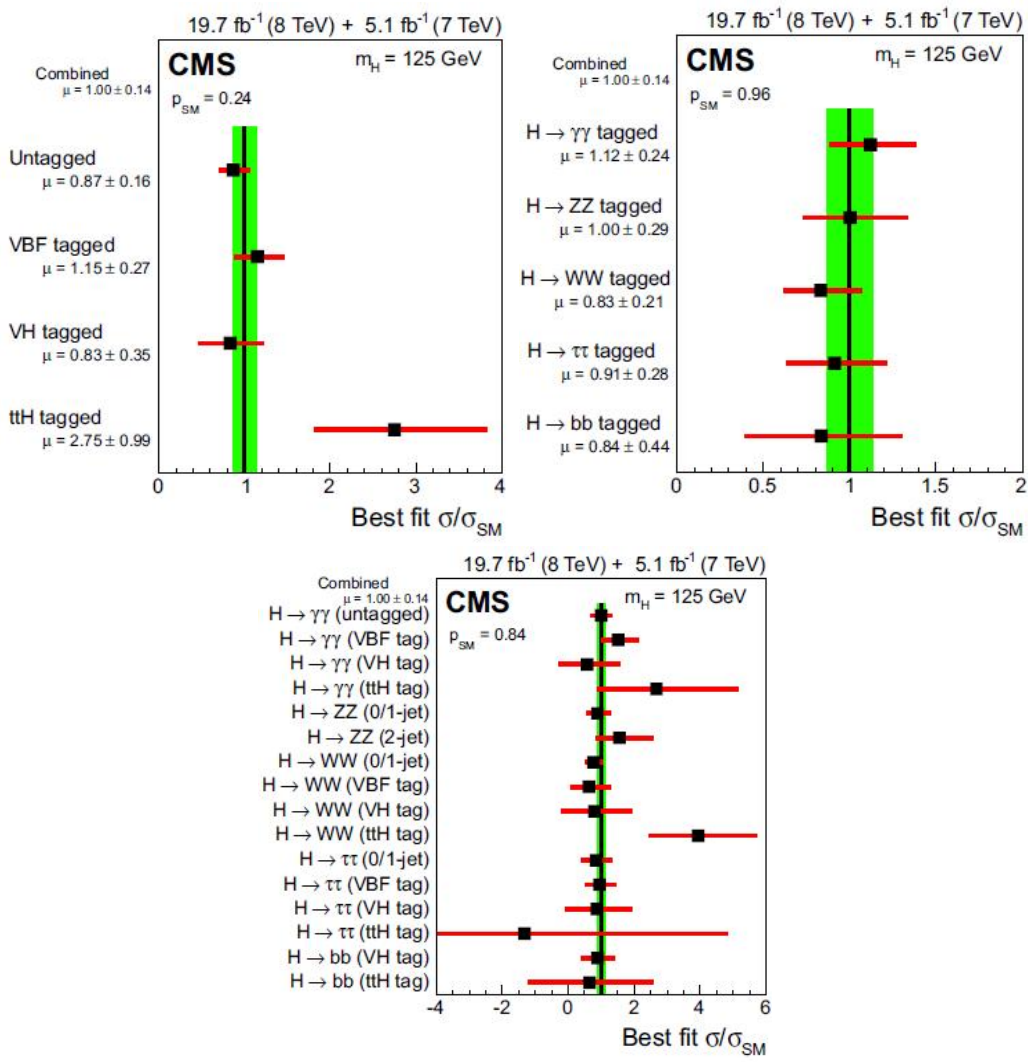

Figure 8. Signal strength for different production (top left), decay modes (to right) and combinations grouped by decay modes and tags of particular production mechanisms (bottom). The horizontal bars indicate the total uncertainty including both the statistical and systematic errors. The vertical green band shows the overall uncertainty on the signal strength. 


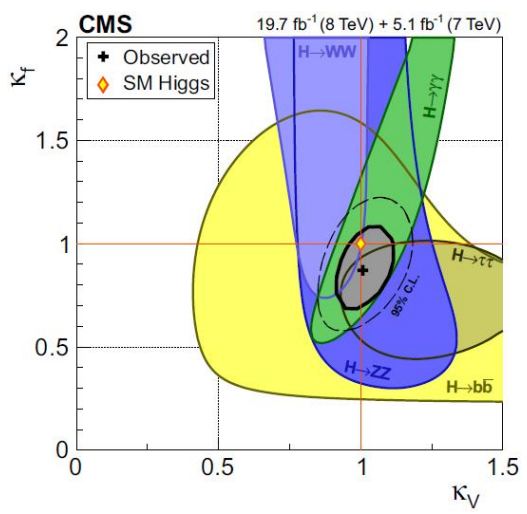

Figure 9. Contours of the $68 \%$ C.L. regions for individual channels and for the combination (full line). The cross corresponds to the best fit and the dashed line represents the 95\% C.L. contour. The SM expectation is indicated by the diamond.

\section{First results at $13 \mathrm{TeV}$}

In this section the first results obtained by CMS with the data collected in 2015, at the center-of-mass energy of $13 \mathrm{TeV}$, are presented. The dataset corresponds to a total luminosity ranging from $2.3 \mathrm{fb}^{-1}$ to $2.8 \mathrm{fb}^{-1}$ depending on the final state. All results are preliminary.

The presence of the Higgs boson peak has been searched in the $H \rightarrow Z Z^{*} \rightarrow 4 \ell$ [22] and in the $H \rightarrow \gamma \gamma$ [23] final states. In the former, there is an indication of the signal with a significance of $2.5 \sigma$ corresponding to $\mu=0.82_{-0.43}^{+0.57}$. The energy dependence of the fiducial cross section, considering $\sqrt{s}=7,8$ and $13 \mathrm{TeV}$ data is compatible, within the large uncertainty, with the SM expectation as shown in Fig. 10. For the diphoton final state analysis [23], the observed (expected) significance with respect to the background only hypothesis is $1.7 \sigma(2.7 \sigma)$ corresponding to $\mu=0.69_{-0.42}^{+0.47}$. The weighted sum of the contributions of all categories is shown in Fig. 11.
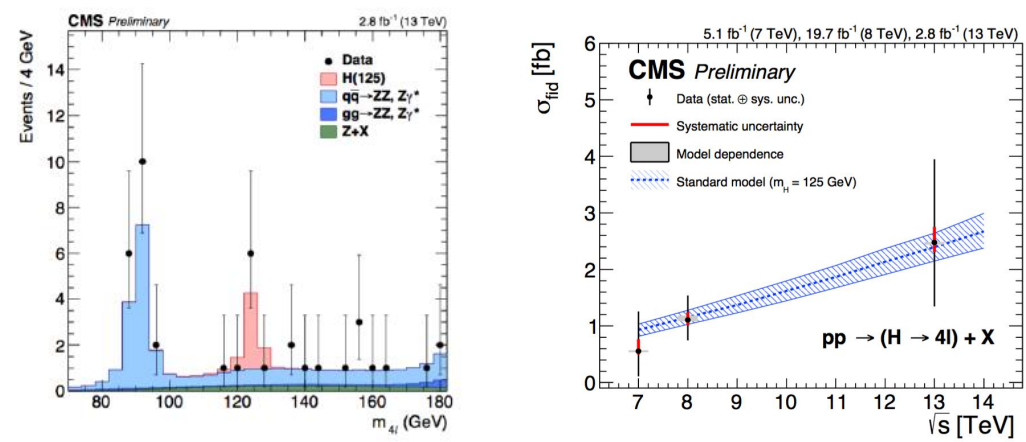

Figure 10. Invariant mass distribution of four lepton final state at $13 \mathrm{TeV}$ center-of-mass energy (left). The measured fiducial cross-section as a function of the center-of-mass energy (right).

Several analyses of production/decay channels successfully finalised using the RUN 1 dataset have been repeated with the first data at higher energy. For the $H \rightarrow W W$ decay, only the e $\mu$ final state has been considered [24], at a preliminary stage, in order to reduce the large Drell-Yan background 


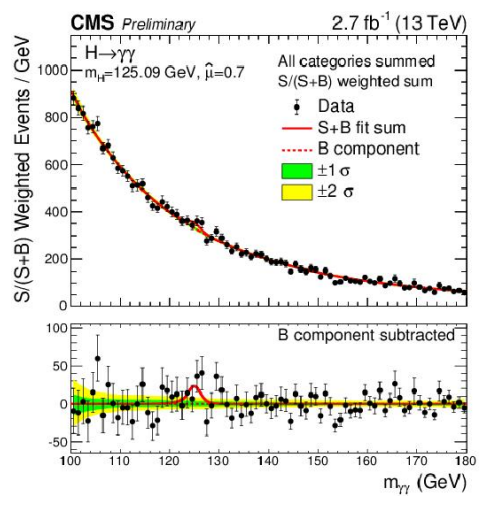

Figure 11. Weighed invariant mass distribution of diphoton final state at $13 \mathrm{TeV}$ center-of-mass energy.

present in the same-flavour dilepton events. Following the same strategy as in RUN 1 analysis, the observed (expected) significance with respect to the background only hypothesis is $0.7 \sigma(2.0 \sigma)$. No evidence of signal has been found in the 2015 dataset for the VBF production of $H \rightarrow b \bar{b}$ decay [25]. However, this negative result has been combined with $8 \mathrm{TeV}$ data result obtaining a global significance of $1.2 \sigma$ corresponding to a signal strength $\mu=1.3_{-1.1}^{+1.2}$ for $m_{H}=125 \mathrm{GeV}$.

Given the particular importance of $t \bar{t} H$ production, several searches have been accomplished for (i) (di)leptonic top decay associated to $H \rightarrow b \bar{b}$ [26], (ii) top tagged $H \rightarrow \gamma \gamma$ decay [23], (iii) multilepton final states [27]. Within the available dataset, none of the searches has found a signal evidence and the combined signal strength is $\mu=0.15_{-0.81}^{+0.95}$ [28].

\section{Implications}

The results obtained by CMS can be summarised as follows [29]:

- the $125 \mathrm{GeV}$ particle is in the range preferred by fits to SM precision measurements;

- it is a scalar so it is a BEH-like boson;

- according to SM, at that mass several final states are accessible;

- it is coupled to bosons and fermions;

- the measured production/decay properties are compatible with the SM expectations at that mass;

- no other states from additional Higgs doublets have been found despite several dedicated searches have been performed.

The above does not prove that the discovered particle is the SM Higgs boson since lightest states of more complex Higgs sectors may have similar properties. In addition it is worth to notice that the value $m_{H}=125 \mathrm{GeV}$ is within the SUSY-accepted range. More precise measurements are necessary to clarify the situation.

\section{Future perspectives}

In a short time scale, the 2016 LHC run is proceeding well and the CMS is taking data with a good efficiency. Furthermore, the production cross sections at $\sqrt{s}=13 \mathrm{TeV}$ increase with respect to $8 \mathrm{TeV}$ 
by a factor $\sim 2.2$ for gluon fusion and by a factor $\sim 3.9$ for $t \bar{t} H$ production. These conditions, at the end of 2016, would allow to obtain a relevant improvement on the measured values, with statistical uncertainties reduced by more than a factor two.

In a middle time scale the perspectives of the LHC program have to be considered. Following the plans, the machine should deliver $300 \div 350 \mathrm{fb}^{-1}$ at $\sqrt{s}=13 \mathrm{TeV}$ or $14 \mathrm{TeV}$. The expected CMS precision on the Higgs couplings [30], with an integrated luminosity of $300 \mathrm{fb}^{-1}$ at $\sqrt{s}=14 \mathrm{TeV}$, are $6-8 \%$ for $\kappa_{\gamma}, \kappa_{g}, \kappa_{W}, \kappa_{Z}, \kappa_{\tau}$, about $13 \%$ for $\kappa_{b}$ and $15 \%$ for $\kappa_{t}$. As shown in the left panel of Fig. 12, in an optimistic scenario with a reduction of a factor two of the theoretical uncertainties which constitutes the dominant part of systematic errors, the expected uncertainties on the Higgs couplings would range from about $5 \%$ for $\kappa_{\gamma}, \kappa_{g}, \kappa_{W}, \kappa_{Z}, \kappa_{\tau}$, to $7-10 \%$ for $\kappa_{b}, \kappa_{t}$. The increase in center-of-mass energy and luminosity gives an opportunity to observe heavy $H$ states as foreseen by MSSM or to extend the excluded region in $m_{A}-\tan \beta$ plane. If the discovered particle is the lightest CP-even state $h$, values of $m_{A}$ smaller than 230-260 GeV will be excluded for all $\tan \beta$ values [31].

In a longer time scale the High Luminosity LHC program would give the ultimate opportunity for CMS to contribute to the Higgs sector. In the right panel of Fig. 12, the estimated precision on the coupling modifiers for an assumed luminosity of $3000 \mathrm{fb}^{-1}$ is shown. The contribution from theoretical errors is more relevant in this case. A high luminosity program gives also an unique chance to observe rare channels as the decay $H \rightarrow \mu \mu$, or the Higgs boson pair production which is related to the Higgs self-coupling $\lambda$.
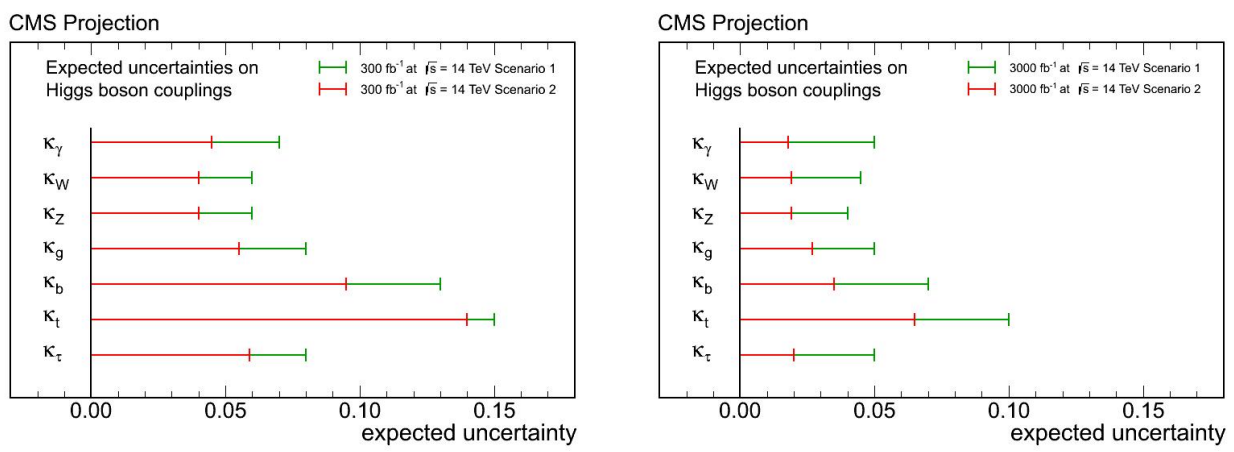

Figure 12. Estimated precision on the measurements for coupling modifiers for a SM-like Higgs boson at a center-of-mass energy of $14 \mathrm{TeV}$ and a dataset with integrated luminosity of 300 (left) and $3000 \mathrm{fb}^{-1}$ (right). Two scenarios for systematic uncertainties are considered (see text for details).

\section{Conclusions}

The discovery of the BEH particle has been not only one of the most important results in particle physics but also one of the most relevant scientific achievements. The discovery requires several further actions that will engage the HEP community for several years to try to give an answer to the following questions [32]:

- is the new particle really the SM BEH Boson?

- is it an elementary particle? or...

- is it a composite particle? 
- is it natural?

- are there other Higgs fields?

- is it really responsible for the mass of all elementary particles?

and also

- is it at the origin of the matter-antimatter asymmetry?

- does it have a role in the inflationary expansion of the Universe?

To obtain a lot of more precise measurements, as expected in the long LHC and High Luminosity LHC programs, is the unique way to answer to the previous questions.

\section{References}

[1] F. Englert and R. Brout, Phys. Rev. Lett. 13, 321 (1964), doi:10.1103/PhysRevLett.13.321.

[2] P. W. Higgs, Phys. Lett. 12, 132 (1964), doi:10.1016/0031-9163(64)91136-9.

[3] P. W. Higgs, Phys. Rev. Lett. 13, 508 (1964), doi:10.1103/PhysRevLett.13.508.

[4] G. S. Guralnik, C. R. Hagen and T. W. B. Kibble, Phys. Rev. Lett. 13, 585 (1964), doi:10.1103/PhysRevLett.13.585.

[5] P. W. Higgs, Phys. Rev. 145, 1156 (1966), doi:10.1103/PhysRev.145.1156.

[6] G. Aad et al., Phys. Lett. B 716, 1 (2012), doi:10.1016/j.physletb.2012.08.020.

[7] S. Chatrchyan et al., Phys. Lett. B 716, 30 (2012), doi:10.1016/j.physletb.2012.08.021.

[8] G.C. Branco et al., Phys. Rep. 516, 1 (2012), doi:10.1016/j.physrep.2012.02.002.

[9] The ALEPH, DELPHI, L3, OPAL Collaborations, the LEP Electroweak Working Group, Phys. Rep. 532, 119 (2013), doi:10.1016/j.physrep.2013.07.004.

[10] S. Chatrchyan et al., Phys. Rev. D 89, 092007 (2014), doi:10.1103/PhysRevD.89.092007.

[11] V. Khachatryan et al., Eur. Phys. J. C (2014) 74: 3076, doi:10.1140/epjc/s10052-014-3076-z

[12] V. Khachatryan et al., Eur. Phys. J. C (2015) 75:212, doi:10.1140/epjc/s10052-015-3351-7

[13] G. Aad et al., Phys. Rev. Lett. 114, 191803, doi:10.1103/PhysRevLett.114.191803

[14] S. Chatrchyan et al., J. High Energ. Phys. 01096 (2014). doi:10.1007/JHEP01(2014)096

[15] S. Chatrchyan et al., Phys. Rev. D 89, 012003 (2014), doi:10.1103/PhysRevD.89.012003.

[16] V. Khachatryan et al.,Phys. Rev. D 92, 032008 (2015), doi:10.1103/PhysRevD.92.032008.

[17] S. Chatrchyan et al., Nature Phys. 10, 557 (2014), doi:10.1038/nphys3005.

[18] V. Khachatryan et al., J. High Energ. Phys. 09087 (2014). doi:10.1007/JHEP09(2014)087

[19] V. Khachatryan et al., Eur. Phys. J. C (2015) 75:251 doi:10.1140/epjc/s10052-015-3454-1

[20] S. Heinemeyer et al., Handbook of LHC Higgs Cross Sections: 3. Higgs Properties: Report of the LHC Higgs Cross Section Working Group. arXiv:1307.1347. CERN-2013-004 https://twiki.cern.ch/twiki/bin/view/LHCPhysics/CrossSections .

[21] M.J.G. Veltman, Nucl. Phys. B 123, 89 (1977). doi:10.1016/0550-3213(77)90342-X

[22] CMS Collaboration: CMS-PAS-HIG-15-004.

[23] CMS Collaboration: CMS-PAS-HIG-15-005.

[24] CMS Collaboration: CMS-PAS-HIG-15-003.

[25] CMS Collaboration: CMS-PAS-HIG-16-003.

[26] CMS Collaboration: CMS-PAS-HIG-16-004.

[27] CMS Collaboration: CMS-PAS-HIG-15-008.

[28] https://twiki.cern.ch/twiki/bin/view/CMSPublic/TTHCombMoriond2016 
[29] P. Checchia, Int.J.Mod.Phys. A30 (2015) 07, 1530003, doi: 10.1142/S0217751X15300033

[30] CMS Collaboration: CMS NOTE-13-002,arXiv:1307.7135.

V. Khachatryan et al.,CERN-LHCC-2015-10.

[31] I. M. Lewis, arXiv:1308.1742.

[32] INFN, CSN1: Proposal for a long term strategy for accelerator based experiments, Phys. Ser. 60 (2015) pp. 1-291. 